\title{
Pharmacist's Role in Improving Medication Safety for Patients in an Allogeneic Hematopoietic Cell Transplant Ambulatory Clinic
}

\author{
Lina Ho, Keith Akada, Hans Messner, John Kuruvilla, Janice Wright, and Jack T Seki
}

\begin{abstract}
Background: Patients undergoing allogeneic hematopoietic cell transplantation (allo-HCT), supported by complex drug regimens, are vulnerable to drug therapy problems (DTPs) at interfaces of care after discharge from hospital and may benefit from timely pharmacy interventions and education.
\end{abstract}

Objective: To determine the effect on medication safety of, as well as potential barriers to, incorporating a pharmacist in the multidisciplinary team of an allo-HCT clinic.

Methods: Two pharmacists rotated to attend the allo-HCT clinic of a tertiary care, university-affiliated cancer centre between January and June 2010 (coverage for 1 of 3 clinic days per week). For every patient who was seen by a pharmacist, all discharge medications were reconciled from the inpatient ward to the clinic. The pharmacists' primary task was to perform medication reconciliation and to identify and resolve DTPs. The pharmacists also provided medication education to patients and pharmacy consultations to clinic staff. Working with the outpatient pharmacy, the pharmacists helped to clarify prescriptions and drug coverage issues. Medication discrepancies identified and interventions performed by the pharmacists were recorded and were later graded for clinical significance by a panel of clinicians. Patient and staff satisfaction surveys were conducted at random during the study period. Barriers to the flow of patient care and other operational issues were documented.

Results: The 2 pharmacists saw a total of 35 patients over 100 visits. They identified a total of 50 medication discrepancies involving 17 (49\%) of the patients and 70 DTPs involving 23 (66\%) of the patients. Thirty-one of the 70 DTPs resulted directly from a medication discrepancy. Twenty (95\%) of the 21 unintentional medication discrepancies and $7(70 \%)$ of the 10 undocumented intentional medication discrepancies were graded as clinically significant or moderately significant. Satisfaction surveys completed by patients and clinic staff yielded positive responses supporting pharmacists participation.

Conclusions: Pharmacists working as part of the multidisciplinary team identified and resolved medication discrepancies, thereby improving medication safety at the allo-HCT clinic.

Key words: medication reconciliation, medication discrepancy, drug therapy problems, medication safety, allogeneic hematopoietic cell transplantation, ambulatory clinic

\section{RÉSUMÉ}

Contexte : Les patients subissant une greffe allogénique de cellules souches hématopoïétiques (GACSH), appuyée par un traitement médicamenteux complexe, sont vulnérables aux problèmes pharmacothérapeutiques lors de changement de milieu de soins après avoir reçu leur congé de l'hôpital et pourraient tirer profit d'interventions et de conseils pharmaceutiques en temps opportun.

Objectif : Déterminer les répercussions sur la sécurité des médicaments de la participation d'un pharmacien et les obstacles potentiels à sa participation à l'équipe multidisciplinaire d'une unité clinique de GACSH.

Méthodes : Deux pharmaciens se sont relayés pour se joindre à l'équipe de l'unité clinique de GACSH d'un centre de cancérologie tertiaire affilié à une université, entre janvier et juin 2010 (participation à une des trois journées par semaine d'ouverture de l'unité clinique). Pour chaque patient quil a rencontré, le pharmacien a comparé les médicaments prescrits au départ de l'hôpital au schéma pharmacothérapeutique adopté à l'unité de GACSH. La principale tâche des pharmaciens était d'effectuer un bilan comparatif des médicaments et de détecter et de résoudre tout problème pharmacothérapeutique. Les pharmaciens devaient également fournir des conseils sur les médicaments aux patients et des consultations au personnel de l'unité clinique. En collaboration avec la pharmacie externe, le pharmacien aidait à clarifier les ordonnances et à préciser les modalités de remboursement des médicaments. Les divergences médicamenteuses relevées par les pharmaciens et les interventions effectuées par ceux-ci ont été consignées, puis dans un second temps classées par un panel de cliniciens selon leur importance clinique. Des sondages sur la satisfaction des patients et du personnel ont été effectués au hasard pendant la période de l'étude. Les obstacles au bon déroulement des soins aux patients et d'autres problèmes de fonctionnement ont été cernés et consignés.

Résultats : Les deux pharmaciens ont vu 35 patients en tout au cours de 100 visites. Ils ont détecté un total de 50 divergences médicamenteuses touchant $17(49 \%)$ des patients et 70 problèmes pharmacothérapeutiques touchant $23(66 \%)$ des patients. Trente-et-un de ces 70 problèmes pharmacothérapeutiques étaient directement attribuables à une divergence médicamenteuse. Vingt (95\%) des 21 divergences non intentionnelles et $7(70 \%)$ des 10 divergences intentionnelles non consignées ont été classées comme étant significatives ou modérément significatives sur le plan clinique. Les sondages sur la satisfaction remplis par les patients et le personnel de l'unité ont dégagé des réponses favorables à la participation des pharmaciens. 
Can J Hosp Pharm 2013;66(2):110-117
Conclusions : Les pharmaciens qui ont participé à l'équipe multidisciplinaire ont détecté et résolu des divergences médicamenteuses, améliorant ainsi la sécurité des médicaments à l'unité de GACSH.

Mots clés : bilan comparatif des médicaments, divergence médicamenteuse, problèmes pharmacothérapeutiques, sécurité des médicaments, greffe allogénique de cellules souches hématopoïétiques, unité de soins ambulatoires

[Traduction par l'éditeur]

\section{INTRODUCTION}

$\mathrm{M}$

ost cancer care delivery in the Canadian health care system takes place in the ambulatory setting. As the clinical acuity and treatment complexity increases for patients with hematologic malignancies, there is a high risk of medication-related adverse events and medication discrepancies. At interfaces of patient care, such as admissions and discharges, medication errors are prone to occur because of gaps in information transfer.

Medication reconciliation is a formalized process that has been demonstrated to identify significant numbers of discrepancies and reduce adverse drug events when it is implemented at interfaces of patient care. ${ }^{1}$ The effectiveness of this approach lies in the application of specific skills in interviewing patients to ascertain the best possible medication history (BPMH) and reconcile it against physician's orders in a timely manner. Electronic clinical tools have also been developed to facilitate the transfer of patient information, including BPMH, at interfaces of patient care. ${ }^{2}$ The benefits of medication reconciliation have been well documented in the inpatient hospital setting at admission and discharge and in the preadmission surgical clinic. $^{3-6}$ In a study performed at the Mayo Clinic, the implementation of medication reconciliation resulted in complete elimination of medication discrepancies on admission and a $42 \%$ reduction in the mean number of medication discrepancies at discharge per patient. ${ }^{3}$

Medication reconciliation in the ambulatory care setting is less well studied. However, it may be equally if not more important in this setting because patients' medications are typically changed at each clinic visit on the basis of clinical presentation. Furthermore, medications may be modified at other health care facilities between clinic visits (e.g., upon admission to a local hospital or during a visit to the family physician). Although patients' presenting signs and symptoms may be managed during these one-time visits, medications prescribed at general practice sites may not adequately address the needs of patients who are receiving care through specialty programs. As such, patients are vulnerable to medicationrelated problems due to drug-drug interactions, potential discrepancies, etc.
Patients who have undergone allogeneic hematopoietic cell transplantation (allo-HCT) represent a high-risk group that must be followed at frequent clinic visits. Their medication therapy fluctuates quickly, and the potential for medication discrepancies is high. In addition, they are supported by complex drug regimens involving chemotherapy and immunosuppressive agents, and errors involving these medications can result in potentially severe adverse events. For these reasons, incorporation of a pharmacist as part of the multidisciplinary team was proposed for the allo-HCT clinic at the Princess Margaret Cancer Centre in Toronto, Ontario, with the idea that implementation of medication reconciliation could improve patient safety and outcomes by reducing errors that arise from incomplete documentation and transfer of medication information.

\section{METHODS}

As an initiative to promote safer medication practices and improve the flow of prescription processing at the allo-HCT clinic at the Princess Margaret Cancer Centre, research funding was secured for a pilot project that allocated 0.2 full-time equivalent of pharmacist time (i.e., 1 day per week) to the clinic over a 6-month period (January to June 2010). For half of this time ( 0.5 day per week), the pharmacist was available for direct patient-related care; this represented coverage for onesixth of the clinic's 3 days per week. The remaining half-day was devoted to indirect patient care duties (e.g., gathering and recording data, communication with and sign-over to the inpatient and outpatient pharmacies). Two pharmacists alternated to attend the allo-HCT clinic. One of the pharmacists (L.H.) had general hospital residency training plus 2 years of experience in inpatient malignant hematology (i.e., leukemia, lymphoma, myeloma, allo-HCT, and autologous stem cell transplant). The other pharmacist (K.A.) had close to 20 years of experience in malignant hematology. At the time of the project, about 80 patients per year underwent transplantation through the allo-HCT program.

After transplantation and before discharge from the allo-HCT ward, all patients received medication teaching by the ward pharmacist. A copy of the discharge medication schedule and the inpatient pharmacy care plan document 
(concise information about the patient, including laboratory testing, demographic characteristics, preadmission medical and medication history, drug coverage, conditioning regimen, identification and resolution of drug therapy problems [DTPs] while in hospital) were forwarded to the clinic pharmacist for follow-up. Before each clinic day, the pharmacist obtained a patient appointment roster and, for selected patients on the roster, reviewed the dictation notes recorded by the discharging physician in the hospital's electronic patient record (EPR).

Patients who had undergone transplantation less than 3 months earlier were the primary target population for this medication safety initiative. Most of these patients were in a clinically acute state: their hematopoietic system might not have fully recovered, and they often required frequent and close monitoring. Although the condition of patients who were beyond 3 months after transplantation was more stable, a small number of these patients had acute and/or chronic complex medication issues for which pharmacy referral was also requested. The intended number of patient visits for each half-day clinic was 5 to 10 (the variation being due to unpredictable patient flow in the clinic and variable duration of individual patient interviews). On average, each patient interview took 15 to $30 \mathrm{~min}$, but some took longer. The total number of patients booked on any given clinic day ranged from 30 to 35; therefore, the pharmacist could see at most one-third to one-quarter of the patients on any given day.

Upon arrival, each patient was interviewed by the clinic pharmacist to obtain the most up-to-date BPMH. For patients making their first clinic visit after discharge, medication reconciliation was carried out by comparing the BPMH obtained at the clinic with the discharge medication schedule prepared by the ward pharmacist and/or the medication list in the dictated electronic discharge summary. For patients making subsequent visits, medication reconciliation was performed by comparing the BPMH against the medication list dictated in previous electronic clinic notes. Medication discrepancies and DTPs identified by the pharmacist were discussed with clinic nurses and physicians before they assessed the patient and were resolved after the patient assessment was completed. Any changes in drug therapy were recorded, and a revised medication schedule was printed for the patient. At the end of the clinic visit, the pharmacist educated the patient about changes to existing medications and any new medications that were to be started.

To expedite the processing of prescriptions, they were screened and clarified at the clinic, with prescribers in close proximity. They were then faxed immediately to the hospital's outpatient pharmacy department to reduce waiting time for patients. For example, the outpatient pharmacy could start preparing the prescriptions while patients were receiving systemic therapy or inhalational treatments or were undergoing bone marrow biopsy or diagnostic imaging. The pharmacist forwarded a copy of the patient's updated medication list to the outpatient pharmacy for MedsCheck billing.* The pharmacist was also available in the clinic for assessment and management of pain and for other types of medication consultations, such as alternative routes of medication administration, treatment of sleeping disorders, and recommendations for anti-infectives, as requested by other members of the health care staff. The pharmacist also addressed drug information questions concerning side effects, availability of dosage forms, dosage adjustments, and eligibility for drug coverage.

The pharmacist entered each patient's data, including DTPs and medication discrepancies, in an electronic spreadsheet. Each DTP identified was classified into 1 of 7 categories. Each medication discrepancy identified was classified as unintentional, undocumented intentional, or documented intentional. The unintentional and undocumented intentional discrepancies were independently graded for clinical significance by a panel of clinicians, consisting of a pharmacist (K.A.), a nurse (J.W.), and a physician (H.M.), all of whom were working in the clinic. ${ }^{4,67}$ This method of assessing the degree of clinical impact of medication discrepancies was adapted from Kwan and others. ${ }^{6}$ Clinically significant, moderately significant, and nonsignificant medication discrepancies were defined as having probable, possible, or unlikely potential, respectively, to cause discomfort and/or clinical deterioration for patients if no actions were taken to identify and address them. At the end of each clinic day, progress notes were recorded in the individual patients' profiles to facilitate follow-up of specific issues at subsequent clinics, such as therapeutic drug monitoring, efficacy and tolerability of drug therapy changes, and unresolved drug coverage issues.

Patient and staff satisfaction surveys were conducted at random throughout the 6-month service period.

\section{RESULTS}

Over the 6-month period of the pilot project, the 2 pharmacists saw a total of 35 patients over the course of 100 patient visits. Twenty-eight of the patients were seen in 3 or more visits. During an estimated $104 \mathrm{~h}$ spent by the pharmacists on

\footnotetext{
*The MedsCheck program is a one-on-one interview between the pharmacist and the patient to review the patient's prescription and nonprescription medications. It is funded by the Ontario Government through the Ontario Drug Benefit Health Network System. A comprehensive drug review list, which is dated and authorized with the pharmacist's and the patient's signatures, is submitted to the Ontario Drug Benefit Program for billing. Each initial "MedsCheck Annual" is reimbursed for Can $\$ 60$ and each subsequent "MedsCheck Followup" is reimbursed for Can $\$ 25$.
} 
direct patient care activities ( $4 \mathrm{~h} /$ week for 26 weeks), 50 medication discrepancies and 70 DTPs were identified and resolved. Thirty-one of the 70 DTPs resulted directly from a medication discrepancy. Physicians and nurse practitioners accepted 99\% of the pharmacists' recommendations. The pharmacists addressed 34 drug information questions, from both patients and clinic staff, and provided 39 medication schedules to patients with billing for MedsCheck. The measurable end points are summarized in Table 1.

One or more medication discrepancies were identified for 17 (49\%) of the 35 patients. Of the 50 medication discrepancies identified, 21 (42\%) were unintentional discrepancies and $10(20 \%)$ were undocumented intentional discrepancies. These 31 discrepancies resulted in subsequent identification and resolution of a variety of DTPs, which are categorized in Table 2. Of the 21 unintentional medication discrepancies, 20 (95\%) were independently graded as clinically significant or moderately significant by the panel of clinicians (pharmacist, nurse, and physician). Of the 10 undocumented intentional discrepancies, $7(70 \%)$ were graded as clinically significant or moderately significant. The remaining 19 (38\%) discrepancies were classified as documented intentional discrepancies that were identified at the time of the medication reconciliation interviews. These discrepancies were attributable to breakdowns in communications, as follows. A pharmacist was present in the clinic only one half-day of the 3 weekly clinic days, on Tuesdays; as such, medications ordered during other clinic days (the Monday before or the Friday of the previous week) were identified as discrepancies at the Tuesday visit. These intentional medication changes were documented in the EPR by the prescribers but were not listed in the medication

\section{Table 1. Summary of Measurable End Points}

\begin{tabular}{|c|c|c|}
\hline Variable & $\begin{aligned} \mathrm{Nc} \\
\text { No. }(\%)\end{aligned}$ & $\begin{array}{l}\text { or } \\
\text { of Patients }\end{array}$ \\
\hline Patients and visits & & \\
\hline No. of patients & 35 & \\
\hline No. of visits & 100 & \\
\hline No. of patients with $\geq 3$ visits & 28 & \\
\hline Medication discrepancies & & \\
\hline No. of medication discrepancies identified & 50 & \\
\hline $\begin{array}{l}\text { No. (\%) of patients with } \geq 1 \text { medication } \\
\text { discrepancies }\end{array}$ & 17 & (49) \\
\hline Drug therapy problems & & \\
\hline No. identified & 70 & \\
\hline No. resulting from medication discrepancy & 31 & \\
\hline $\begin{array}{l}\text { No. }(\%) \text { of patients with } \geq 1 \text { drug } \\
\text { therapy problems }\end{array}$ & 23 & $(66)$ \\
\hline Other outcomes & & \\
\hline No. (\%) of recommendations accepted & $88 / 89$ & (99) \\
\hline $\begin{array}{l}\text { No. of medication schedules provided } \\
\text { to patients }\end{array}$ & 39 & \\
\hline No. of drug information questions addressec & 34 & \\
\hline
\end{tabular}

schedule most recently prepared for the patient by the pharmacist. Following the patient interview, all types of outstanding discrepancies identified in the EPR or from the patient's current medication list were consolidated and further reconciled with the patient and the prescriber.

One or more DTPs were identified for 23 (66\%) of the 35 patients. Of the 70 DTPS identified and resolved by the pharmacists, 31 resulted directly from a medication discrepancy, as described above; the other 39 DTPs were independent of medication reconciliation. The $70 \mathrm{DTPs}$ were subdivided into 7 categories (see Table 3). In 15 (21\%) of the DTPs, drug therapy was required for a clinical indication but had not been prescribed (e.g., prophylactic acyclovir was not restarted after discontinuation of ganciclovir treatment), whereas 11 DTPs $(16 \%)$ involved continuing drug therapy that was no longer necessary (e.g., posaconazole prophylaxis was continued beyond 100 days post-transplant in a patient with no graftversus-host disease and no history of fungal infection). Ten DTPs (14\%) involved drug therapy that was not effective (e.g., a patient was taking $3 \mathrm{mg}$ of lorazepam for insomnia without good effect). Nineteen DTPs (27\%) involved inappropriate drug dosages (dosage was too high in 4 cases [e.g., supratherapeutic phenytoin level in a patient experiencing dizziness] and too low in 15 cases [e.g., inadequate pain control]). Five DTPs (7\%) involved adverse drug reactions (e.g., renal toxicity from vancomycin which subsequently required discontinuation), and 10 DTPs (14\%) involved inappropriate administration (e.g., cyclosporine taken before blood sample was drawn for measurement of trough level or patient not remaining in upright position after taking a bisphosphonate).

Satisfaction surveys from both clinic staff and patients yielded positive responses supporting inclusion of a pharmacist as part of the multidisciplinary team (Appendices 1 and 2). Unresolved barriers related to patient flow and operational issues limited the efficiency of distribution and retrieval of the surveys. The 13 completed patient surveys that were returned represented just under $40 \%$ of clinic patients. The results indicated that patients valued the pharmacist as a drug information and education resource and believed that the pharmacist acted on their behalf in addressing drug-related needs. On a scale of 1 to 5 (where $1=$ strongly disagree and 5 = strongly agree), all patient respondents either agreed or strongly agreed that the pharmacist was an essential part of the allo-HCT clinic team and wanted the pharmacy service in the clinic to continue (average score 4.8/5). From the clinic staff's perspective (based on the 7 staff surveys returned), the pharmacist was valued as an educational resource for both staff and patients. In addition, staff respondents felt that the pharmacist was useful for drug information inquiries, especially regarding drug toxicity and interactions. Similar to patient respondents, all clinic staff respondents either agreed or strong- 


\section{Table 2. Characterization of Discrepancies}

\begin{tabular}{|c|c|c|c|}
\hline \multirow[b]{2}{*}{ Type of Discrepancy } & \multicolumn{3}{|c|}{ No. of Discrepancies } \\
\hline & Unintentional & $\begin{array}{l}\text { Undocumented } \\
\text { Intentional }\end{array}$ & Total \\
\hline Omission: patient was taking a medication that was not documented & 3 & 7 & 10 \\
\hline Commission: patient was not taking a medication that was documented & 1 & 1 & 2 \\
\hline Incorrect dosage & 7 & 1 & 8 \\
\hline Incorrect frequency & 6 & 0 & 6 \\
\hline Incorrect duration of therapy & 3 & 1 & 4 \\
\hline Incorrect drug & 2 & 0 & 2 \\
\hline Total & $21 *$ & 10 & $31 *$ \\
\hline
\end{tabular}

*Total for this column is less than the sum of data above because one unintentional discrepancy involved both incorrect dosage and incorrect frequency.

ly agreed that the pharmacist was an integral part of the clinic team and should become a regular member of the team (average score 4.6/5).

\section{DISCUSSION}

Previous researchers have demonstrated that medication reconciliation can prevent clinically significant medication discrepancies. ${ }^{89}$ Although medication reconciliation is a standard of care in the inpatient wards at the Princess Margaret Cancer Centre (at admission, transfer, and discharge), it has not yet been uniformly adopted in our ambulatory clinics, including the allo-HCT clinic. One of the reasons may be the lack of a standardized practice model for the ambulatory medication reconciliation process. In addition, the inability of the electronic pharmacy system to interface with the hospital EPR system makes it difficult for the pharmacy to provide other health care staff with the most up-to-date medication reconciliation information.

The primary objective of this pilot project was to determine the impact of clinical pharmacy services (with a focus on medication reconciliation and resolution of DTPs) on medication safety at the allo-HCT clinic, which serves one of the most complex patient populations at this institution. These patients' medication regimens are changed drastically after transplantation, and the drugs involved are high-risk medications such as chemotherapy and immunosuppressive agents with narrow therapeutic windows. In addition, readmissions are common for this patient population, either directly from the clinic back to the inpatient wards, from home to local hospitals, or via transfer from local emergency departments to the inpatient wards. The involvement of multiple patient care interfaces may result in high rates of medication errors and omissions through gaps in information transfer.

Previous studies have shown that medication discrepancies occur frequently on hospital discharge, with rates ranging from $39.6 \%$ to $56.3 \%$. $^{1,4,5}$ Consistent with these studies, $49 \%$ of the patients seen in this pilot study had at least one medication
Table 3. Frequency of 7 Drug Therapy Problems Identified from Interventions

\begin{tabular}{lc} 
Type of Problem & No. of Problems \\
\hline Drug therapy required but not prescribed & 15 \\
Unnecessary therapy & 11 \\
Therapy not effective & 10 \\
Dose too high & 4 \\
Dose too low & 15 \\
Adverse drug reaction & 5 \\
Non-adherence & 10
\end{tabular}

discrepancy. Of the 31 unintentional and undocumented intentional medication discrepancies identified in this study, 27 (87\%) were clinically significant or moderately significant, consistent with a study conducted at The Moncton Hospital, in which 91\% (90/99) of medication discrepancies were clinically significant or very significant. ${ }^{1}$ These data show the high rate of clinically significant medication discrepancies among patients visiting ambulatory clinics. This pilot project was consistent with current literature on medication reconciliation in terms of showing a need for medication reconciliation in the ambulatory care setting, beyond hospital discharge.

Ruder and others ${ }^{10}$ described the effect of clinical pharmacy services in a US oncology ambulatory clinic. This retrospective analysis involved a diverse patient population with a variety of disease sites and stages of disease who were seen over a 2-year period. Interventions were categorized as either drug-related (medication reconciliation, dosing, and management and prevention of adverse drug effects) or consultative (drug information questions, patient visits, and patient education). These interventions are similar to the ones provided by the pharmacy service at the allo-HCT clinic described here, where the pharmacists were able to identify and resolve DTPs, perform medication reconciliation, provide patients with updated mediation schedules, and offer drug information and consultations to clinic staff. In the current study, the pharmacists identified an average of 2 DTPs per patient and made a total of 89 recommendations, 88 of which were accepted by 
prescribers. These data, together with the positive survey results, provided encouraging support that the inclusion of a clinical pharmacist in a multidisciplinary team at an ambulatory oncology clinic can be highly beneficial for both staff and patients.

This project was conducted in collaboration with the hospital's outpatient pharmacy, where the majority of prescriptions generated at the allo-HCT clinic are filled. This collaboration represented another opportunity for the clinic pharmacists to enhance patient and staff satisfaction, by taking seamless care services to a new level. Before sending prescriptions to the outpatient pharmacy, the clinic pharmacist screened them for errors and drug coverage issues, clarifying any issues immediately with prescribers in the clinic. Prescriptions were then faxed to the outpatient pharmacy for timely processing. Feedback from clinic staff indicated that when the clinic pharmacist was present, they received fewer telephone calls and pages from the outpatient pharmacy for order clarifications. With this added efficiency, the outpatient pharmacy was also able to operate without unnecessary interruptions. Ultimately, this benefited patients because of a reduction in wait time for their medications and resolution of drug coverage issues. For example, the clinic pharmacists identified 12 instances in which a drug was prescribed for which the patient had no drug coverage (in some cases, the prescription was modified to an alternative drug or cancelled if it was determined that the drug was not essential; in cases where the drug was essential and there was no acceptable alternative, the outpatient pharmacy agreed to carry the balance forward while waiting for approval of relevant drug coverage applications). A previous unpublished internal review at the institution's outpatient pharmacy showed that an average of 30 min was needed to clarify any problematic prescription with a prescriber. Another aspect of this collaboration was the use of medication schedules prepared by the clinic pharmacist for MedsCheck billing to the Ministry of Health by the outpatient pharmacy. We propose that when the clinic pharmacist position is firmly established, providing a consistent amount of MedsCheck billing, the revenue generated can be used to fund the clinic pharmacist position, which would make it a selfsustaining program.

This study had several limitations. Because some patients were seen multiple times by the pharmacist during the study period for follow-up, the number of medication discrepancies and DTPs identified may be lower than in other studies of medication reconciliation in which patients were usually seen only once (e.g., at admission or at discharge). In addition, although pharmacist interventions led to the resolution of numerous medication discrepancies and DTPs, the study was not designed to measure any patient outcome end points, such as readmission rates or reductions in morbidity and mortality.
Nonetheless, as we have shown, the pharmacist was uniquely suited to provide management and follow-up of acute drugrelated issues, such as pain management. In a few cases, we found it necessary to do additional, unplanned telephone follow-up with the patients at home, which avoided potential hospital readmissions. Pharmacists were also referred to care for patients who called the clinic for ongoing medication issues, which freed up physicians for more urgent cases. These outcomes suggest that pharmacists were able not only to provide clinical services but also to affect overall patient outcomes and workflow.

As a pilot project, this study had some logistical issues that must be considered when the program is further developed in the future. On busy clinic days, patient flow sometimes became congested because some of the medication reconciliation interviews were lengthy; as a result, the clinic fell behind schedule. On other occasions, the pharmacist was planning to meet with a patient for follow-up on a particular issue, only to learn that the patient had already left the clinic. To minimize frustration and maximize efficiency, a systematic and consistent sequence of patient flow needs to be established and must remain transparent to both staff and patients. Another challenge was the difficulty of printing and distributing medication schedules on busy clinic days. There was no standardized fixed venue to store these documents (e.g., charts, patient profiles, electronic space). At the time of this study, the institution's clinics were in the process of switching to paperless charting. When medication schedules were misplaced or went missing and clinic staff relied instead on the dictation from previous visits (a non-reconciliation process), we found that the same medication discrepancies would be perpetuated to subsequent clinic visits. A centralized, electronic patient medication profiling system should be developed and should be accessible to all staff.

\section{CONCLUSIONS}

With the implementation of medication reconciliation services in the ambulatory allo-HCT clinic, a new standard of patient care was defined at our institution. This study has shown that a pharmacist, working as part of the multidisciplinary team, can improve medication safety for patients in the allo-HCT clinic. We believe that pharmacists' clinical interventions as presented in this study have exemplified a number of key performance indicators. Although it was difficult to measure the impact of pharmacy clinical activities on patient outcomes and the described metrics focused primarily on operational functions, the clinic pharmacists contributed to relevant, measureable end points. Operational issues such as systematic patient flow and centralized electronic medication profiling must be addressed to enable the pharmacists to work efficiently and effectively within the team. 
References

1. Nickerson A, MacKinnon NJ, Roberts N, Saulnier L. Drug-therapy problems, inconsistencies and omissions identified during a medication reconciliation and seamless care service. Healthc Q. 2005;8 Spec No.:65-72.

2. Cesta A, Bajcar JM, Ong SW, Fernandes OA. The EMITT Study: development and evaluation of a medication information transfer tool. Ann Pharmacother. 2006;40(6):1074-81.

3. Varkey P, Cunningham J, O'Meara J, Bonacci R, Desai N, Sheeler R. Multidisciplinary approach to inpatient medication reconciliation in an academic setting. Am J Health Syst Pharm. 2007;64(8):850-4.

4. Wong JD, Bajcar JM, Wong GG, Alibhai SM, Huh JH, Cesta A, et al. Medication reconciliation at hospital discharge: evaluating discrepancies. Ann Pharmacother. 2008;42(10):1373-9.

5. Vira T, Colquhoun M, Etchells E. Reconcilable differences: correcting medication errors at hospital admission and discharge. Qual Saf Health Care. 2006;15(2):122-6.

6. Kwan Y, Fernandes OA, Nagge JJ, Wong GG, Huh JH, Hurn DA, et al. Pharmacist medication assessments in a surgical preadmission clinic. Arch Intern Med. 2007;167(10):1034-40.

7. Lee J, Leblanc K, Fernandes O, Huh JH, Wong GG, Hamandi B, et al. Medication reconciliation during internal hospital transfer and impact of computerized prescribed order entry. Ann Pharmacother. 2010; 44(12):1887-95.

8. Bond CA, Raehl CL. Clinical pharmacy services, pharmacy staffing, and hospital mortality rates. Pharmacotherapy. 2007;27(4):481-93.

9. Kaboli PJ, Hoth AB, McClimon BJ, Schnipper JL. Clinical pharmacists and inpatient medical care: a systematic review. Arch Intern Med. 2006;166(9):955-64.

10. Ruder AD, Smith DL, Madsen MT, Kass FH 3rd. Is there a benefit to having a clinical oncology pharmacist on staff at a community oncology clinic? J Oncol Pharm Pract. 2011;17(4):425-32.

Lina Ho, BScPhm, ACPR, is with the Department of Pharmacy, Princess Margaret Cancer Centre, University Health Network, Toronto, Ontario.

Keith Akada, BScPhm, is with the Department of Pharmacy, Princess Margaret Cancer Centre, University Health Network, Toronto, Ontario.

\section{Appendix 1. Patient Satisfaction Survey $(n=13)$}

\section{Questions}

Q1 Pharmacists in the clinic reviewed my medication schedule ensuring what I take is appropriate.

Q2 Pharmacists are valuable as a patient education resource and have helped me to understand the medications I take.

Q3 Pharmacists act on patients' behalf in addressing their health care needs (e.g., drug coverage, expedite processing and delivery of my medications)

Q4 Pharmacists are an essential part of the allo-HCT clinic team. I would like to see this service continued.

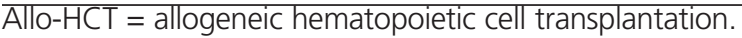

\section{Response options}

\begin{tabular}{ll}
\hline 1 & Strongly disagree \\
3 & Disagree \\
4 & Neither agree nor disagree \\
5 & Agree \\
\hline
\end{tabular}

Hans Messner, MD, PhD, FRCPC, is with the Allogeneic Blood and Marrow Transplant Program, Division of Medical Oncology and Hematology, Princess Margaret Cancer Centre, University Health Network, Toronto, Ontario.

John Kuruvilla, MD, FRCPC, is with the Allogeneic Blood and Marrow Transplant Program, Division of Medical Oncology and Hematology, Princess Margaret Cancer Centre, University Health Network, Toronto, Ontario.

Janice Wright, RN-EC, MS, was, at the time of this study, with the Allogeneic Blood and Marrow Transplant Program, Division of Medical Oncology and Hematology, Princess Margaret Cancer Centre, University Health Network, Toronto, Ontario. She is now with the inpatient rehabilitation program at the Hotel Dieu Shaver Health and Rehabilitation Centre, St. Catharines, Ontario.

Jack T Seki, BScPhm, PharmD, is with the Department of Pharmacy, Princess Margaret Cancer Centre, University Health Network, and the Leslie Dan Faculty of Pharmacy, University of Toronto, Toronto, Ontario.

Competing interests: Lina $\mathrm{Ho}$ and Keith Akada each received an honorarium from the Canadian Blood and Marrow Transplant Group for copresenting the results of this study at a conference in April 2012 in Toronto, Ontario. None declared for other authors.

\section{Address correspondence to:}

Lina Ho

Department of Pharmacy

Princess Margaret Cancer Centre, Room 3-301

610 University Avenue

Toronto ON M5G 2M9

e-mail: lina.ho@uhn.ca

Acknowledgements: We thank all of the staff at the Princess Margaret Cancer Centre allo-HCT clinic. We thank Karen Chuk and her staff in the outpatient pharmacy for their collaboration. We thank Triyu Vather for editing assistance in advance of submission.

\section{Survey results}

\begin{tabular}{lcccc} 
Respondent & Q1 & Q2 & Q3 & Q4 \\
\hline 1 & 5 & 5 & 5 & 5 \\
2 & 5 & 5 & 5 & 5 \\
3 & 4 & 5 & 4 & 4 \\
4 & 5 & 5 & 5 & 5 \\
5 & 5 & 5 & 5 & 5 \\
6 & 5 & 5 & 5 & 5 \\
7 & 5 & 4 & 3 & 4 \\
8 & 5 & 5 & 5 & 5 \\
9 & 4 & 4 & 4 & 5 \\
10 & 5 & 5 & 5 & 5 \\
11 & 5 & 5 & 5 & 5 \\
12 & 4 & 4 & 3 & 5 \\
13 & 5 & 5 & 5 & 5 \\
\hline Average & 4.8 & 4.8 & 4.5 & 4.8 \\
\hline
\end{tabular}




\section{Appendix 2. Staff Satisfaction Survey $(n=7)$}

\section{Questions}

Q1 Presence of pharmacists in the clinic improved the quality of care of patients.

Q2 Pharmacist is valuable as patient educator.

Q3 Pharmacist is valuable as staff teaching resource.

Q4 Pharmacist is useful for consultation regarding adverse effects/toxicity of drugs.

Q5 Pharmacist is useful for consultation regarding drug interactions.

Q6 Pharmacist is helpful to me for making medication decisions based on efficacy.

Q7 Pharmacist is helpful for selection of drugs based on efficacy.

Q8 Pharmacist is helpful for making medication decisions based on drug pricing.

Q9 Pharmacist is an integral part of clinic team.

Q10 Pharmacist should be a regular member of the team.

\section{Response options}

1 Strongly disagree

2 Disagree

3 Neither agree nor disagree

4 Agree

5 Strongly agree

\section{Survey results}

\begin{tabular}{lllllllllll} 
Respondent & Q1 & Q2 & Q3 & Q4 & Q5 & Q6 & Q7 & Q8 & Q9 & Q10 \\
\hline 1 & 5 & 5 & 5 & 5 & 5 & 5 & 5 & 5 & 5 & 5 \\
2 & 5 & 5 & 5 & 5 & 5 & 5 & 4 & 5 & 5 & 5 \\
3 & 5 & 5 & 5 & 5 & 5 & 5 & 4 & 4 & 5 & 5 \\
4 & 4 & 4 & 4 & 5 & 5 & 5 & 4 & 4 & 5 & 5 \\
5 & 4 & 4 & 4 & 4 & 4 & 4 & 4 & 4 & 4 & 4 \\
6 & 3 & 4 & 4 & 4 & 4 & 3 & 3 & 3 & 4 & 4 \\
7 & 3 & 4 & 4 & 4 & 4 & 3 & 3 & 3 & 4 & 4 \\
\hline Average & 4.1 & 4.4 & 4.4 & 4.6 & 4.6 & 4.3 & 3.9 & 4.0 & 4.6 & 4.6 \\
\hline
\end{tabular}

